\title{
Swearing and Its Impact on Physical and Mental Health of Clinical Nurses
}

\author{
Sale Zhang ${ }^{1}$, Yan Sun ${ }^{2, *}$, Ying $\mathrm{Li}^{1}$, Yu Zhang ${ }^{1}$, Huaifen $\mathrm{Ma}^{1}$, Yingtao $\mathrm{Li}^{1}$ \\ ${ }^{1}$ Xi'an Peihua University, Xi'an, 710125, China \\ ${ }^{2}$ Xi'an Jiaotong University, Xi'an, 710061, China \\ ${ }^{*}$ Corresponding author. Email: sunyanwanwan@163.com
}

\begin{abstract}
The paper analyzes the definition and causes of swearing, and its impact on the physical and mental health of clinical nurses, discuss the coping strategies to encourage a deeper understanding of workplace violence in hospitals and pay more attention to the physical and mental health of nurses and provide evidence for managers to introduce effective measures to prevent workplace violence. Keywords: swearing, clinical nurse, workplace violence, verbal aggression, physical and mental health, literature review
\end{abstract}

\section{INTRODUCTION}

Medical facilities are considered one of the places with the highest incidence of violence [1]. Violence in the medical field means that medical personnel are abused, threatened, or physically attacked before, during, or after performing medical activities in health care institutions at all levels, such as hospitals, community health service centers, or other locations, resulting in their safety obvious or implicit challenges to happiness and health [2]. Studies have shown that verbal aggression is the most common form of violence in medical settings [3], and further studies by foreign scholars have found that "swearing" is the most common form of verbal aggression [4]. "Swearing" corresponds to the Chinese word "Li Ma"[5].

Researchers have conducted a number of studies on the incidence, causes, and countermeasures of violence in medical settings. At the same time, their understanding of verbal aggression has become more and more clear, but there was less research on the incidence of swearing, its causes, and its impact on the physical and mental health of medical staff. Overseas scholars have analyzed the incidence of swearing[6,7], the causes of swearing[8], and swearing in medical places[9]; while domestic scholars' research on swearing has focused on linguistics, such as the function of swearing in expression and comparative analysis of the connection and difference between English and Chinese verbal aggression[10-11]. The purpose of this study is to analyze swearing based on the existing literature and to provide a basis for clinical nurses to fully and correctly understand swearing and take reasonable measures.

\section{DEFINITIONS}

\subsection{Workplace Violence}

The World Health Organization's definition of workplace violence refers to incidents of violence in the workplace that are work-related or work-related violence that endanger the safety or health of workers, including "physical violence, verbal aggression, threatening, sexual harassment, racial harassment, and psychological violence"[12].

\subsection{Verbal Aggression}

It is a form of violence intended to harm or threaten others' self-concept through language, which can often cause psychological pressure on others [13].

\subsection{Swearing}

"Lì" as a word is not common in people's daily life, but as a way of communicating or expressing emotions is closely related to people's life and work [10]. The definition of "Lì" in The Sixth Edition of the Modern Chinese Dictionary is swearing, which can be grouped into verbal swearing and verbal rhetoric (that is, abusive words)[14]. Some scholars have more specifically defined swearing as the use of offensive language to cause shock or anger in others [15]. In addition, some studies have suggested that swearing has a positive effect in some situations. For example, some people think that swearing is helpful to vent their negative emotions, and humorous swearing can maintain relationships with others. It can also play a role in maintaining the therapeutic relationship between doctors and patients in medical places $[2,10]$. 


\subsection{Swearwords}

Swearwords are words that are offensive in a certain cultural context and are often used to express strong emotions and attitudes [8]. Some scholars in China have pointed out that abusive words not only carry information but also a way to express emotions [16].

\section{BACKGROUNG}

\subsection{The Universality of Swearing}

Abusive behaviors are common in people's daily life. Studies have found that swearing has gradually become a new language form in people's daily communication since the 1960s [17]; some scholars pointed out that human has abusive behavior since we have language, and most of us have had abusive behavior [11]. In 2012, a questionnaire survey of 100 college students in China found that all participants had abusive behaviour [18].

\subsection{Reasons for Swearing}

Some people want to make others feel pain through swearing [10] and some people think that abusive behavior can show their own personality, or just abusive because of rebellious and herd mentality [2]. Some researchers have clarified the positive role of swearing from the perspective of social psychology. They believe that swearing can help people relieve stress, vent their negative emotions and frustration [19], improve team cohesion, improve the sense of humor, and also make abusers' language sounds more convincing [16].

\subsection{Swear Words}

There are many forms of swear words. The common classifications in foreign studies are religion-related (such as Jesus, heaven, hell, damn), and sex-related (such as fuck, cunt), race-related (e.g. nigger), excretion related (e.g. shit, piss), etc. [8]. In China, it is often related to family or elders (such as fucking, bitch-raised), face-related (such as shame, shameless), and physical or mental retardation (such as: stupid), animal-related (such as pig, livestock), etc. [2,19]. With the development of the economy and the integration of multiple cultures, more and more abusive words from English have appeared in our country. The common ones are shit, pig, fuck, etc. Due to the differences in religious beliefs, some religions Swear words (such as God, Christ, heaven) are not offensive to the Chinese. In recent years, some new forms of abusive words have gradually emerged, such as JR (slut), SB (silly), BT (perverted), BC (idiot), etc. [18]; the letter "B" is becoming more and more popular among young people. For example, "2B" is used to describe a person being stupid, "NB" (Niu Bi) refers to a person who is very powerful. In Chinese culture, "B" (Bi) refers to the female genitalia; fuck referred swear words; numbers are often used as swear words, such as two, two hundred and

\section{CONNECTIONS AND DIFFERENCES BETWEEN SWEARING AND VERBAL AGGRESSION}

\subsection{Comparison of Incidence in Medical Places}

Although swearing is considered to be the most common form of verbal aggression[22], the research on swearing in medical places is very limited, which may be due to the complex and diverse forms of verbal aggression, often including irony, unjustified accusations, public ridicule, threats, and sexual harassment and so on [23]. A study conducted in 2019 found that almost half participants $(n=384)$ had experienced physical violence and almost all had experienced verbal aggression, with a total of $40.7 \%$ doctors and $34.3 \%$ midwives have experienced physical violence, $94.6 \%$ doctors and $85.4 \%$ midwives have experienced verbal aggression [24]. In China, although there have been no reports about swearing in medical places, researchers have begun to classify and discuss verbal aggression in depth. Xiangjun Zou and others found 1,398 participants from 15 different levels of general hospitals in Shaanxi Province during 2014-2015, 67.7\% had experienced workplace violence, $66.2 \%$ had experienced verbal aggression, $8.7 \%$ had experienced physical attacks, and $2.7 \%$ had experienced sexual violence [25]. In another study, a questionnaire survey of 246 undergraduate nursing students in clinical practice in Bin Zhou found that $56(22.76 \%)$ were ridiculed, 51 $(20.73 \%)$ were threatened, 47 (19.11\%) family members of the patients have insulting behavior, the proportion of sarcasm between the nurses was 42 (17.07\%) [26]. These studies have pointed out that swearing is the most important form of verbal aggression. It seems similar to foreign research findings, but there is a big difference between "abusive" and "swearing". The sixth edition of the Modern Chinese Dictionary points out that abusive and swearing are synonymous [14], but abusive refers to abusive language, with a strong insult and aggressiveness, and swearing includes not only abusive behavior but also plays a positive role in communication. This indicates that swearing should be distinguished from other forms of verbal aggression [16]. Researchers should conduct in-depth and comprehensive research on swearing.

\subsection{Impact on Nurses' Physical and Mental Health}

A comparative study found that verbal aggression in the workplace can seriously affect nurses' physical and mental health, often manifested as sleep disorders, low mood, and physical and mental fatigue [27], and some nurses may become anxious, irritable, hostility, some even diarrhea [28], and all these adverse reactions may lead to the decline of nurses' ability to work, loss of sympathy for some aggressive patients, decline in job satisfaction and the occurrence of turnover [23]. Similar to the impact of 
verbal aggression, an investigation found that swearing is a serious problem in nursing work. In the research, an open-ended questionnaire found that the nurse felt very frustrated after describing that she had been abused. Feeling angry, some people even feel that abusing hurts themselves no less than the physical pain caused by physical violence. All participants $(n=84)$ said that they would choose to stay away from the abuser after being abused, which also means the quality of care will be affected [28]. The difference between the two is that although research has shown that abuse in medical settings is often related to stress and aggression [29], An Australian scholar pointed out that abuse is an effective communication method for some patients to express their feelings. In some cases, the abusive behavior of medical staff can even maintain a relationship with the patient [30]. For example, some patients will use abusive language as a signal to change the relationship from unfamiliar to familiar. Facilitate the establishment of therapeutic trust relationships with each other. The results of this study also provide evidence for further research on the effects of abuse on nurses' physical and mental health.

\subsection{Coping Strategies}

A study in China found that $75.42 \%(n=1174)$ responders in workplace chose to explain patiently even suffered violence, $57.40 \%$ of the respondents chose to tolerate, $31.76 \%$ respondents chose to ask for security, $21.98 \%$ of them chose to ask colleagues, $21.37 \%$ of them chose to ask for leadership, and only $10.69 \%$ of them chose to "alarm"[31]. Some scholars found that avoidance or patience is not an effective method to control workplace violence. However, due to the nature of nursing work, nurses often do not have extra time and energy to deal with it [32]. Some scholars have found that improving hospital security measures, strengthening education and training for nurses, and improving communication skills between nurses and patients can effectively prevent and control workplace violence in the emergency department [28], and help nurses adopt a positive attitude in handling workplace violence [30]. However, some scholars hold the opposite view that training of nurses will change their attitudes, perceptions and reporting on workplace violence, and will increase the incidence of workplace violence [33]. A study points out that to effectively respond to abusive behaviors in the workplace, first of all, hospitals should implement a "zero tolerance" policy on swearing, and medical staff can refuse to provide medical services to abusers; second, nurses are the main victims of abuse, leaders' support is very important to them. A safe working environment not only enables nurses to maintain a relaxed and happy working mood but also directly affects the relationship between nurses and patients. Finally, swearing is even a tendency for violence. The precursors of physical violence should be given enough attention, and timely response measures will avoid more serious damage [22,[30]. On the one hand, Robertson et al. [34] surveyed 56 native English speakers (average age 23) and 39 native Japanese speakers (average age 21), found that the abuser's tolerance to pain was regardless of cultural background, this finding may mean that sometimes abusive behaviors of patients are not aimed at others but to alleviate their own pain, which also provides a new way of thinking for nurses' coping strategies after being abused in the workplace.

\section{SUMMARY}

In summary, verbal aggression is the most common form of violence in the medical workplace, and swearing as the most common form of verbal aggression should be studied further. The findings of domestic and foreign scholars on the incidence of verbal aggression, the impact on nurses' physical and mental health, and their coping strategies are basically the same; while the research on swearing is not comprehensive, foreign scholars have started to pay attention to medical places. The swearing phenomenon in China also provides a new direction for Chinese researchers.

Based on the above analysis, it is not difficult to find that swearing in medical workplaces may adversely affect the physical and mental health of nurses, and also have an important role in the doctor-patient relationship. Verbal aggression is mixed with swearing, and nurses' awareness of swearing is particularly important. Some researchers have found that [35-36] if a nurse is attacked by a patient, it will affect the nurse's attitude towards the patient when he encounters the same situation next time, which also suggests that sometimes the nurse cannot effectively perform nursing operations for the patient in order to avoid being attacked by the patient, which will lead to a decline in the quality of care.

If the nurse can correctly understand the abusive behavior of the patient, realize that sometimes the swearing of the patient may be due to his or her illness, uncertainty about the disease and treatment, and emotions such as anxiety, anger, and fear rather than targeting the nurse himself. Understanding of swearing will help maintain the nurse-patient relationship and reduce its impact on the physical and mental health of nurses. Based on the existing literature, this article analyzes the connection and differences between swearing and verbal aggression in medical settings, hoping to provide inspiration for in-depth research and provide a basis for managers to formulate effective measures to prevent workplace violence.

\section{ACKNOWLEDGMENT}

This work was supported by Xi'an Social Science Foundation ((NO. 19y83).

\section{REFERENCES}

[1] Kowalenko T, Cunningham R, Sachs C J, et al. Workplace Violence in Emergency Medicine: Current Knowledge and Future Directions [J]. The Journal of Emergency Medicine, 2012, 43(3):523-531. 
[17] Vaattovaara J, Peterson E. Same old paska or new shit? On the stylistic boundaries and social meaning potentials of swearing loanwords in Finnish [J]. Ampersand, 2019, 6:100057.

[18] Chu qunwu, Chen xiaguang. Analysis of characteristics and social psychology of abusive language of college students [J] Journal of huzhou normal university, 2012, 34(05):98-102. (In Chinese)

[19] Zhang Yisheng. On lexicalization, marketization and construction of cursing words -- on the expressive function of cursing words in evolution in contemporary Chinese [J]. Contemporary rhetoric, 2010(04):1-13. (In Chinese)

[20] Hong liang. Cursing in modern Chinese literature [J]. Journal of radio and television university (philosophy and social sciences edition), 2016(02):68-73. (In Chinese)

[21] Xu Yong. A study on the causes of homophonic phenomena in new media [J]. Southeast communication, 2019(08):114-116. (In Chinese)

[22] Ramacciati N, Gili A, Mezzetti A, et al. Violence towards Emergency Nurses: The 2016 Italian National Survey-A cross-sectional study[J]. J Nurs Manag, 2019, 27(4):792-805.

[23] Li N, Zhang L, Xiao G, et al. The relationship between workplace violence, job satisfaction and turnover intention in emergency nurses $[\mathrm{J}]$. International Emergency Nursing, 2019, 45:50-55.

[24] Hamzaoglu N, Türk B. Prevalence of Physical and Verbal Violence against Health Care Workers in Turkey $[\mathrm{J}]$. International Journal of Health Services, 2019, 49(4):844-861.

[25] Cai Jianzheng, Wang haifang, Mao Lifen et al. Qualitative research on high-risk situations of workplace violence in nurses [J]. Journal of nursing, 2008, 25(08):1-4. (In Chinese)

[26] Wang Qinghua, Pang Liming, Zhang Fenfen, et al. Investigation on verbal aggression suffered by undergraduate nursing students in a grade a hospital in Bin Zhou in the early stage of clinical practice [J]. Qilu nursing journal, 2016, 22(04):53-54. (In Chinese)

[27] Choi S H, Lee H. Workplace violence against nurses in Korea and its impact on the professional quality of life and turnover intention [J]. J Nurs Manag, 2017, 25(7):508-518.

[28] Guo Jinyu, Sun Hong, Zhou Ying. Investigation of occurrence and influence of verbal violence among medical staff in the emergency department $[\mathrm{J}]$. Nursing Research, 2017, 31(02):217-220. (In Chinese)

[29] Zhang Min. How to carry out prevention and 
control of workplace violence at the hospital level [J]. China nursing management, 2019, 19(04):481-487. (In Chinese)

[30] Stone T E, Mcmillan M, Hazelton M. Swearing: its prevalence in healthcare settings and impact on nursing practice $[\mathrm{J}]$. J Psychiatr Ment Health Nurs, 2010, 17(6):528-534.

[31] Kong Deling, Wang Mingxu, Kong Shuzhen, et al. Investigation and study on workplace violence suffered by medical staff in a third-class first-class hospital in xi 'an [J]. Chinese medical ethics, 2018, 31(01):50-54. (In Chinese)

[32] Pien L C, Cheng Y, Cheng W J. Internal workplace violence from colleagues is more strongly associated with poor health outcomes in nurses than violence from patients and families [J]. Journal of Advanced Nursing, 2018, 75(4):793-800.

[33] Needham I A C H R. The effect of a training course in aggression management on mental health nurses' perceptions of aggression: a cluster randomized controlled trial $[\mathrm{J}]$. International Journal of Nursing Studies, 2015, 42(6):649-655.

[34] Olivia Robertsona S J R R. Swearing as a response to pain: A cross-cultural comparison of British and Japanese participants [J]. Scandinavian journal of pain, 2017(17).

[35] Jef Adriaenssens V D G S. The impact of traumatic events on emergency room nurses: Findings from a questionnaire survey [J]. International Journal of Nursing Studies, 2012, 49(11).

[36] Jiang Shasha, Jiang Zhenghui. Influence of workplace violence on nurses' decision-making ability in nursing ethics $[\mathrm{J}]$. Journal of nursing management, 2015, 15(2):132-134. (In Chinese) 